\title{
Cold vacuum chamber for diagnostics: Instrumentation and first results
}

\author{
S. Gerstl, ${ }^{*}$ R. Voutta, ${ }^{\dagger}$ S. Casalbuoni, ${ }^{\ddagger}$ A. W. Grau, T. Holubek, and D. Saez de Jauregui \\ ANKA, Karlsruhe Institute of Technology, P.O. Box 3640, D-76021 Karlsruhe, Germany \\ R. Bartolini, M. P. Cox, E. C. Longhi, G. Rehm, J. C. Schouten, and R. P. Walker \\ Diamond Light Source, Oxfordshire OX11 ODE, England \\ G. Sikler \\ Babcock Noell GmbH, Alfred-Nobel-Str. 20, 97080 Würzburg, Germany
}

M. Migliorati and B. Spataro

INFN-LNF, Via E. Fermi, 4000044 Frascati, Roma, Italy

(Received 2 July 2014; published 24 October 2014)

\begin{abstract}
For a proper design of the cryogenic layout of superconducting insertion devices it is necessary to take into account the heat load from the beam to the cold beam tube. In order to measure and possibly understand the beam heat load to a cold bore, a cold vacuum chamber for diagnostics (COLDDIAG) has been built. COLDDIAG is designed in a flexible way, to allow its installation in different light sources. In order to study the beam heat load and the influence of the cryosorbed gas layer, the instrumentation comprises temperature sensors, pressure gauges, and mass spectrometers as well as retarding field analyzers with which it is possible to measure the beam heat load, total pressure, and gas content as well as the flux of particles hitting the chamber walls. In this paper we describe the experimental equipment, the installation of COLDDIAG in the Diamond Light Source and selected examples of the measurements performed to show the capabilities of this unique instrument.
\end{abstract}

DOI: 10.1103/PhysRevSTAB.17.103201

PACS numbers: 29.27.-a, 41.75.Ht, 84.71.Ba

\section{INTRODUCTION}

In order to increase the photon flux and the brilliance, synchrotron light sources make use of insertion devices (IDs). Superconducting IDs have higher fields for a given gap and period length compared with the state of the art technology of permanent magnet IDs. One of the still open issues of fundamental importance for the development of superconducting IDs is the knowledge of the beam heat load to the cold vacuum chamber. This is needed for the design of the cooling system of the cryostat that keeps the coils in the superconducting state.

Cold bore superconducting wigglers [1-3] and an undulator [4] installed in different storage rings have been used also to estimate the beam heat load; however, none of those IDs was designed and built to perform beam heat load diagnostics and some of the measured parameters might be affected by significant errors. The resistive wall heating model nicely reproduces the measured values of the beam

\footnotetext{
*stefan.gerstl@kit.edu

†robert.voutta@kit.edu

†sara.casalbuoni@kit.edu
}

Published by the American Physical Society under the terms of the Creative Commons Attribution 3.0 License. Further distribution of this work must maintain attribution to the author $(s)$ and the published article's title, journal citation, and DOI. heat load to the cold beam pipe of the superconducting undulator built by ACCEL Instruments $\mathrm{GmbH}$, Bergisch Gladbach, Germany, at the synchrotron light source ANKA in the so-called low alpha operation mode of the machine, where the electron bunches are shortened to a few ps [5] to generate coherent synchrotron radiation [6]. During the normal user operation mode of ANKA and for the other superconducting cold bore wigglers installed in MAX-II and in the Diamond Light Source (DLS), the estimated values of the beam heat load are higher than the values calculated from synchrotron radiation from the upstream bending magnet and resistive wall heating. During the normal user operation mode of ANKA, a simple model of electron bombardment appears to be consistent with the large variation of beam heat load and of pressure rise values as a function of the average beam current for different gaps $[5,7]$, observed in the cold bore of the superconducting undulator. It has been shown [8] that in order to reproduce the pressure measurements in the ANKA cold bore superconducting undulator, it is necessary to include in the equations of gas dynamic balance, electron stimulated desorption with a shorter decay than the beam lifetime. This implies a very fast avalanchelike growth of the electron flux as a function of beam current, suggesting electron multipacting. Still to be understood is the mechanism responsible for the electron multipacting and the eventual role played by the cryosorbed gas layer. 
The beam heat load measurements performed with cold bore superconducting wigglers and undulators installed in different storage rings are not yet understood. Two dedicated experimental setups, the LBNL-SINAP (Lawrence Berkeley National Laboratory-Shanghai Institute of Applied Physics) calorimeter [9,10] and COLDDIAG [11-13], have been designed and built to measure the beam heat load with high accuracies $<0.1 \mathrm{~W}$ and to help in understanding the beam heating mechanism. Even if both setups are designed to measure the beam heat load, they are nicely complementary. While the LBNL-SINAP calorimeter will allow beam heat load measurements at different gaps, the COLDDIAG has one cold and two warm sections, and it is equipped with additional diagnostics such as retarding field analyzers, pressure gauges, and mass spectrometers to shed light on the eventual role played by the cryogenic layer in the beam heating mechanism. The LBNL-SINAP calorimeter is installed in the Shanghai Light Source [14].

COLDDIAG was installed in November 2011 in the DLS [15]. Because of a mechanical failure at one thermal transition, it was removed after only one week of operation. A redesign of the faulty transition in COLDDIAG allowed reinstallation in August 2012 [16]. A picture of the experimental setup installed in the storage ring of the DLS is shown in Fig. 1. In this paper we describe the cryostat and the instrumentation. Together with beam heat load measurements we present the measured total flux of charged particles impinging on the chamber walls, the influence of a solenoidal field on these particles, as well as the measured pressure and the residual gas content. A second paper will focus on the detailed analysis of the measurements performed during one year of operation at the DLS.

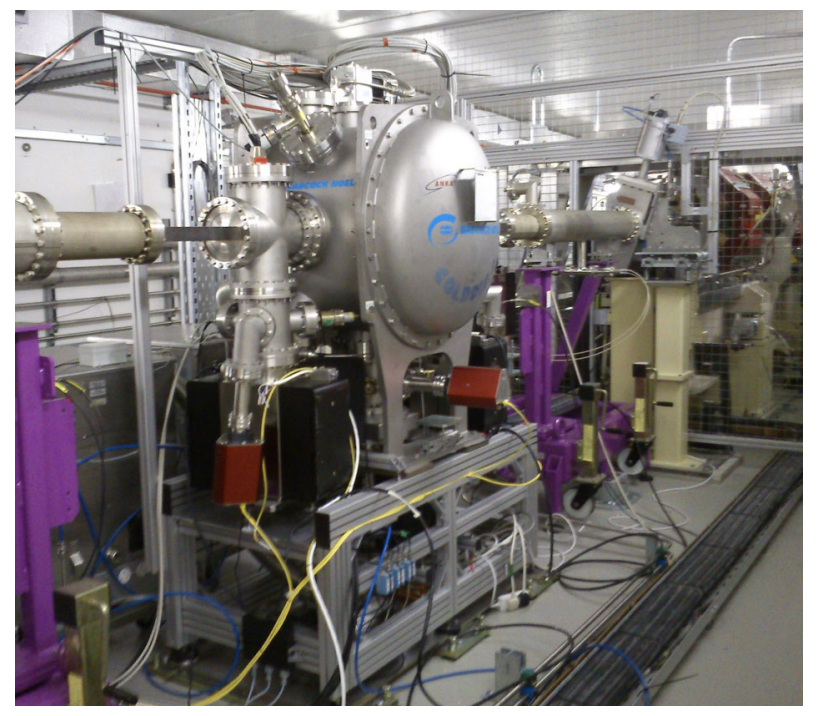

FIG. 1. COLDDIAG installed in the DLS in August 2012.

\section{POSSIBLE BEAM HEAT LOAD SOURCES}

Possible beam heat load sources are synchrotron radiation, rf effects due to geometrical and resistive wall impedance, and electron and/or ion bombardment.

In order to screen insertion devices from the synchrotron radiation produced by the upstream bending magnet, a collimator system is normally installed. However, reflected synchrotron radiation can still shine onto the cold section of the insertion device. The amount of reflected synchrotron radiation hitting the cold chamber walls depends on the geometry of the beam pipe in front of the ID, on the position and shape of the collimator, as well as on the material and on the roughness of the surfaces. An analytical expression of the synchrotron radiation power emitted from the upstream bending magnet directly hitting the upper and lower surfaces of the vertical gap of a downstream chamber is given in Refs. [2,5]. The beam heat load contribution from direct and reflected synchrotron radiation depends linearly on the stored average beam current $I$. It depends on the electron beam energy and on the geometry, that is on the relative position of the bending magnet, the collimator, and the liner. It is however independent on the filling pattern and on the bunch length.

Radio-frequency effects due to geometrical and resistive wall impedance consider the beam heating due to the electromagnetic interaction of the beam with the surrounding chamber. Geometrical impedance is caused by changes of the beam pipe geometry like steps, gaps, tapers, and surface roughness. These are almost unavoidable at the transitions, where tapers, if fingers, and bellows are employed. Even flanges connecting parts with the same aperture contribute to cross section changes due to the finite mechanical accuracy of the manufactured components. Resistive wall impedance is caused by the finite conductivity of a smooth beam pipe. In case of $M$ equi-spaced and equi-populated bunches, the beam heating due to the interaction of the beam with the longitudinal impedance scales, in case of broad band as $I^{2} / M$, while in case of narrow band impedance, it scales as $I^{2}$ [17]. The beam heat load due to rf effects depends also on the bunch length, on the filling pattern, and on the position of the bunch in the vacuum chamber. It does not depend on the beam energy.

Ions and electrons created by ionization and photodesorption, and accelerated against the wall by the passing beam, will also contribute to the beam heat load. The beam dynamics involved is unknown and might be complicated. It is however likely that it is dominated by the beam properties and by the chamber surface characteristics, such as secondary emission yield, photoemission yield, photoemission induced electron energy distribution, etc., which are only partially measured for a cryosorbed gas layer. Since the beam dynamics is unknown we do not know for this source of beam heat load its dependence on the different beam parameters such as filling pattern, beam energy, average stored beam current, bunch length, bunch 
spacing, and number of bunches. Taking this into account, we cannot state that an observed linear or quadratic dependence of the beam heat load on the average beam current $I$ is sufficient to prove that the main contribution to the beam heat load comes from synchrotron radiation or $\mathrm{rf}$ effects, respectively.

\section{THE VACUUM CHAMBER}

COLDDIAG is made up of a cold vacuum chamber located between two warm sections (see Fig. 2), one upstream and one downstream. This allows one to make a direct comparison of the cryogenic and room temperature regions, with and without a cryosorbed gas layer, respectively. In addition it might permit one to observe the influence of synchrotron radiation on the beam heat load. While the warm sections consist of a single volume of ultrahigh vacuum (UHV), the cryostat includes two volumes: the isolation and the beam vacuum (UHV). A DN100 conflat (CF) stainless steel six-way cross separates the two vacua. The electron beam goes through a liner designed to be exchangeable. The first liner, built for the tests in the DLS, is machined from solid copper (also used for thermal stabilization) which is itself plated with $50 \mu \mathrm{m}$ of copper. It has an elliptical cross section similar to the DLS superconducting wigglers with semiaxes $5 \times 30 \mathrm{~mm}$. The resistance at low temperatures in the first few $\mu \mathrm{m}$ of material exposed to the beam is an important parameter that determines resistive wall heating losses. The $50 \mu \mathrm{m}$ of copper plating resembles the surface seen by the electron

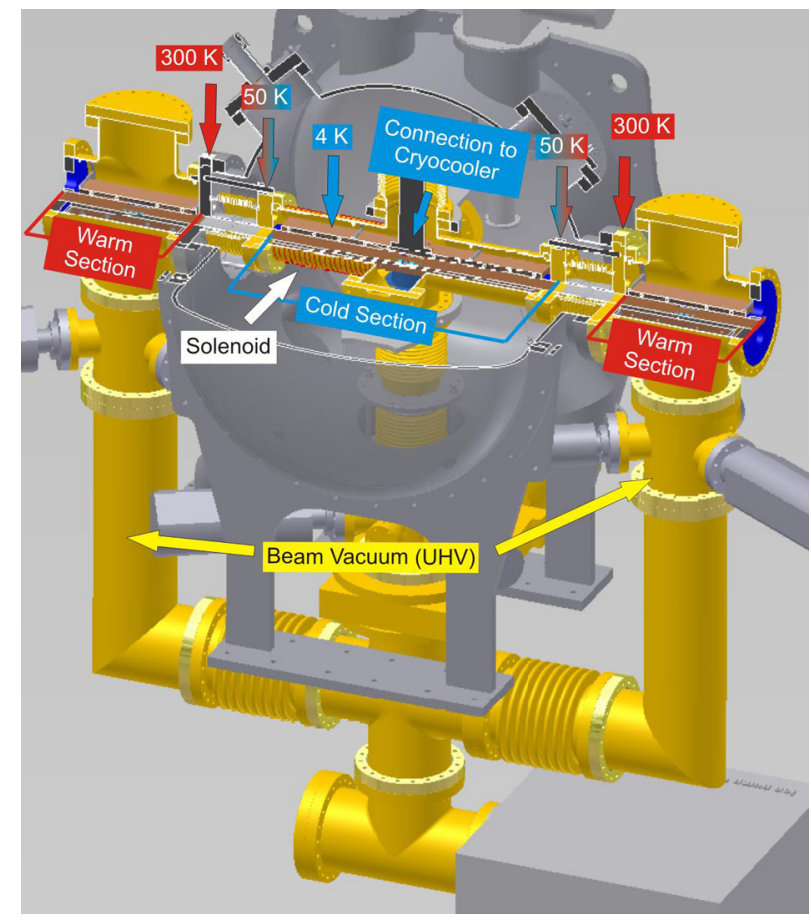

FIG. 2. Overview of the cryostat and the vacuum chamber. beam in the liners used in the ANKA superconducting undulators, made of $300 \mu \mathrm{m}$ stainless steel foil with $30 \mu \mathrm{m}$ of copper plating $[11,18]$. The temperature of the liner in different superconducting IDs ranges from around 4-20 K $[1,4,19]$. In order to simulate the cold bore of superconducting IDs, the liner must be cooled down to reach the above mentioned temperature range. Moreover, to study the beam heat load as a function of the liner base temperature (without beam), it is desirable to operate COLDDIAG with the cold liner reaching about $4 \mathrm{~K}$ in absence of beam, and with the possibility of operating at higher temperatures up to $300 \mathrm{~K}$. The liner base temperature is determined by the quality of the thermal transitions connecting the cold liner to the $50 \mathrm{~K}$ shield. Here and in the following, $4 \mathrm{~K}$ liner and $50 \mathrm{~K}$ shield indicate the liner and the shield connected to the second and first stage of the cryocooler, respectively. The temperatures $4 \mathrm{~K}$ and $50 \mathrm{~K}$ are just indicative. The thermal transition should minimize as much as possible the heat transfer between the two temperature regions, and unavoidably, due to mechanical tolerances, introduce steps upstream and downstream.

Bellows equipped with rf fingers are needed between the $50 \mathrm{~K}$ shield and the $300 \mathrm{~K}$ vessel for the thermal contraction, as the cold parts cool down. The warm copper liners placed upstream and downstream with respect to the cryostat are mechanically connected to the outer beam tube through flanges which minimize the heat exchange. The thermal insulation is needed for the cold and the warm copper liners in order to measure the beam heat load on these three parts of the beam pipe.

For the diagnostic port in the cold section a design based on the COLDEX device [20] was chosen. The diagnostic devices are connected to the cold liner through a warm tube to avoid gas condensation along the path between liner and diagnostic devices. The diagnostic ports in the warm section are a simplified version of the one in the cold section. The geometry of the holes in the liner is a standard DLS design and is the same for all three ports. A huge effort has been made to keep the mechanical tolerances below $10 \mu \mathrm{m}$ along the middle of the cold beam pipe within $\pm 10 \mathrm{~mm}$. In the warm regions the connection of the copper liner to the outer flanges presents steps of about $100 \mu \mathrm{m}$.

\section{A. The cryogenic layout}

COLDDIAG is a cryogen-free system and cooled by one Sumitomo RDK-415D cryocooler, which has a specified cooling power of $35 \mathrm{~W}$ at $50 \mathrm{~K}$ on the warm stage and $1.5 \mathrm{~W}$ at $4.2 \mathrm{~K}$ on the cold stage [21]. This subsection describes the cryogenic layout and the heat intake path from conduction and radiation. Heat transfer by convection is neglected as it is very efficiently suppressed by the insulation vacuum.

A summary of the estimated main contributions to the thermal budget of COLDDIAG is given in Table I. The main contributions from thermal radiation to the heat 
TABLE I. Estimated main contributions to the thermal load budget of COLDDIAG without beam, considering the solenoid on and perfect thermal surface contact at the transitions between 4 and $50 \mathrm{~K}$ at the liner ends.

\begin{tabular}{lcc}
\hline \hline & $300-50 \mathrm{~K}$ & $50-4 \mathrm{~K}$ \\
\hline Radiation & Thermal shield 8 W & Thermal shield 0.06 W \\
& Diagnostic tube 4 W & $\begin{array}{c}\text { Diagnostic tube 0.12 W } \\
\text { Liner ends 0.02 W }\end{array}$ \\
Sum & $12 \mathrm{~W}$ & $0.2 \mathrm{~W}$ \\
Conduction & Thread rods 3 W & Thermal transition \\
& $\begin{array}{l}\text { Stainless steel rods 14 W } \\
\text { rf bellow + fingers 14 W }\end{array}$ & At liner ends 0.4 W \\
& Solenoid at 1 A 8 W & \\
Sum & $40 \mathrm{~W}$ & $0.4 \mathrm{~W}$ \\
Total heat & $52 \mathrm{~W}$ & $0.6 \mathrm{~W}$ \\
\hline \hline
\end{tabular}

budget of the $50 \mathrm{~K}$ stage are given by the radiation from the $300 \mathrm{~K}$ vessel $(\simeq 8 \mathrm{~W})$ to a thermal shield covered with superinsulation, directly thermally connected to the warm stage of the cryocooler, and from the central diagnostics tube $(\simeq 4 \mathrm{~W})$, for a total amount estimated to be about $12 \mathrm{~W}$. The design of this tube (see Fig. 3) is based on the one used for the COLDEX experiment [20] which was installed at the Super Proton Synchrotron at CERN. This tube connects the inside of the cold beam pipe with the residual gas analyzer and pressure gauges. To avoid molecules from getting frozen on their way from the beam pipe to the residual gas diagnostic instruments, this tube needs to be at ambient temperature and has to sit as close as possible to the liner (at a distance of about $1 \mathrm{~mm}$ ) without direct contact. Although the radiated heat from this diagnostics tube is reduced by a shield at floating temperature, this warm insert deposits heating power on both the thermal shield and the cold beam pipe $(\simeq 120 \mathrm{~mW}$ at the $4 \mathrm{~K}$ stage). Together with the radiation from the diagnostic tube, the main contributions to the $4 \mathrm{~K}$ stage due to thermal

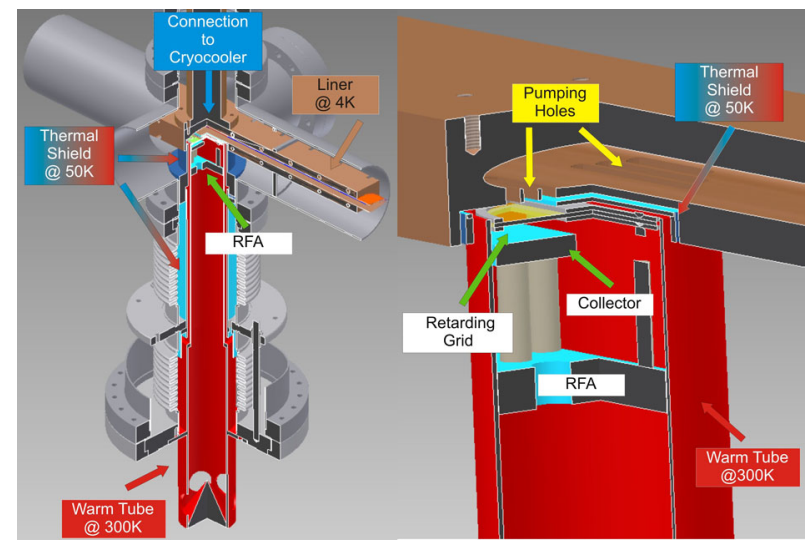

FIG. 3. Drawing of the diagnostics tube. radiation are the elliptical apertures on the liner ends $(\simeq 20 \mathrm{~mW})$ and the $50 \mathrm{~K}$ thermal shield $(\simeq 60 \mathrm{~mW})$, for a total amount of about $200 \mathrm{~mW}$.

The heat budget from thermal conduction is higher than the one from thermal radiation at both stages of the cryocooler. In Fig. 4 the parts contributing to the heat budget due to thermal conduction from room temperature to the $50 \mathrm{~K}$ shield temperature are highlighted in yellow, while the ones from the $50 \mathrm{~K}$ shield to the $4 \mathrm{~K}$ cold liner are in green. The contribution to the heat transfer from ambient temperature to the $50 \mathrm{~K}$ region coming from the TiAl6V4 thread rods with a diameter of $2 \mathrm{~mm}$ and a length of more than $300 \mathrm{~mm}$, supporting the inner cold mass, and from the bellows is about $3 \mathrm{~W}$ in total and therefore negligible. The relevant heat inputs come from the $10 \mathrm{~mm}$ diameter stainless steel guidance rods $(\simeq 14 \mathrm{~W})$ at the rf bellows and the stainless steel rf fingers $(\simeq 14 \mathrm{~W})$, for which the worst case of a perfect thermal contact through the contact surfaces has been assumed. The total heat transfer from ambient temperature to the $50 \mathrm{~K}$ region due to conduction is estimated to be about $32 \mathrm{~W}$. A solenoid, installed to suppress charged particles impinging onto the liner walls, consists of 1920 windings of $1 \mathrm{~mm}$ diameter copper wire wound around the downstream horizontal arm of the cold cross. A current of about $1 \mathrm{~A}$ is needed to produce a longitudinal magnetic field with an on-axis value in the middle of the solenoid of about $10 \mathrm{mT}$. With $1 \mathrm{~A}$ and a resistance of $\simeq 8 \Omega$ at an operation temperature around $50 \mathrm{~K}$ a heat intake of $\simeq 8 \mathrm{~W}$ is estimated.

The total heat transfer from the $50 \mathrm{~K}$ to the $4 \mathrm{~K}$ region is dominated by the thermal transition at the two ends of the cold liner. This will be discussed in the next section. The other inputs coming from the suspension rods $(\simeq 50 \mathrm{~mW})$, the vertical bellows $(\simeq 10 \mathrm{~mW})$, and the horizontal arms of the cold cross $(\simeq 50 \mathrm{~mW})$ are negligible.

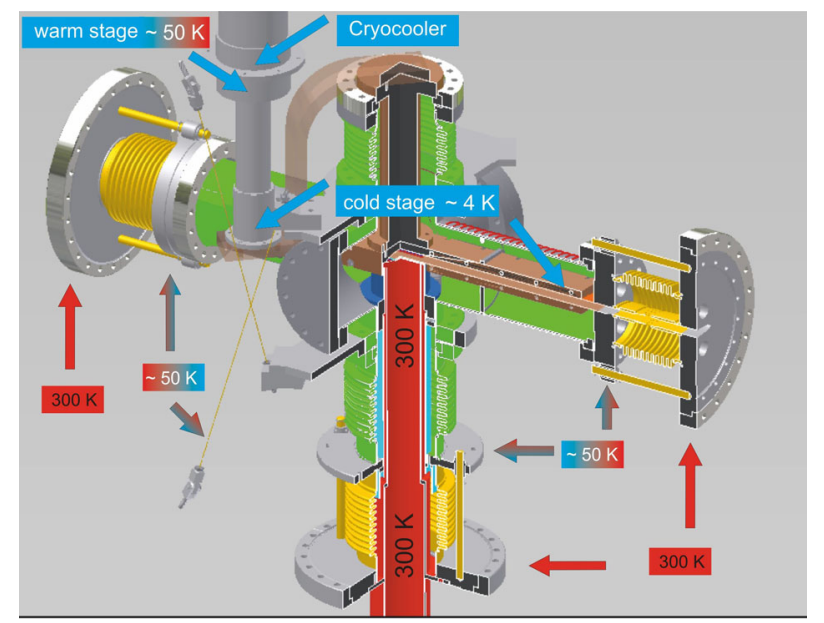

FIG. 4. Highlighted in yellow are the transition from 300 to $50 \mathrm{~K}$, and in green the transitions from 50 to $4 \mathrm{~K}$. 


\section{B. The thermal transition between 4 and $50 \mathrm{~K}$}

To minimize the heat transfer, the transition is made out of a $50 \mu \mathrm{m}$ thin stainless steel foil (see Fig. 5), and covered with $5 \mu \mathrm{m}$ electrodeposited copper to minimize heating from the image currents of the electron beam. For the first installation a $20 \mathrm{~mm}$ long transition sliding inside the flange of the bellows was used. On the colder side it was glued with a ceramic cementlike glue (Aremco Ceramabond 571). For this thermal transition a thermal intake of about $200 \mathrm{~mW}$ each due to conduction was estimated as the worst case, with perfect thermal contact at the surface contacts. The base temperature reached without beam was in this case $4.02 \pm 0.25 \mathrm{~K}$. COLDDIAG was installed in the storage ring of the DLS during a machine shutdown in November 2011. After the first successful tests

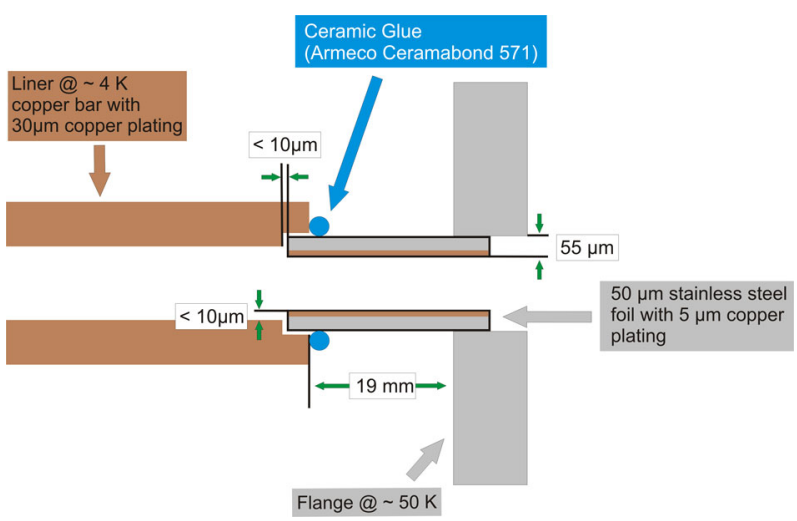

FIG. 5. Thermal transition from rf bellows to the cold liner section.

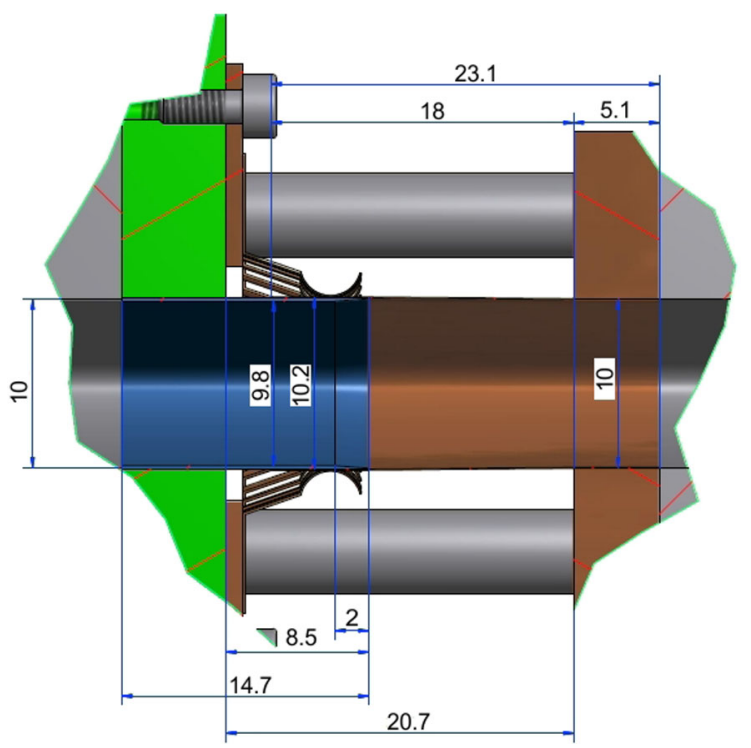

FIG. 6. Drawing of the improved thermal transition foil. The dimensions are all indicated in $\mathrm{mm}$. and measurements with beam, the thermal transitions from the rf bellows flange to the cold liner section bent towards the inner part of the vacuum chamber. It was possible to run the storage ring only by inserting a bump in the orbit of the electron beam. Consequently, COLDDIAG had to be removed after only one week of user beam. The lack of contact pressure, good for minimizing the thermal coupling between the $50 \mathrm{~K}$ shield and the $4 \mathrm{~K}$ liner, made a bad electrical contact on the sliding joint between the transition foil and the rf-bellow flange. This might have caused an increased geometric impedance and consequently a temperature rise followed by a thermal expansion of the transition foil, which bent.

To allow the possibility of a second installation, a new thermal transition mechanically more robust was designed and manufactured. A drawing is shown in Fig. 6. In order to improve the electrical contact, the transition is split into two foils pressed together by copper beryllium springs: a $50 \mu \mathrm{m}$ thin stainless steel foil electroplated with $5 \mu \mathrm{m}$ of copper is soft soldered to the copper liner and slides on a $200 \mu \mathrm{m}$ thick copper foil soft soldered to the rf bellow [22]. To minimize heating effects from a possible step from the thin to the thick foil, the edge of the copper foil is chamfered with a tapering angle of less than $6^{\circ}$. To reduce the electrical resistance of the contacts, only copper to copper transitions are made. The drawback of this more solid design is a better thermal connection between the $50 \mathrm{~K}$ shield and the $4 \mathrm{~K}$ liner, which however still allowed one to reach a low base temperature of $5.28 \pm 0.25 \mathrm{~K}$. A sketch of the cold liner including the transitions from 4 to $50 \mathrm{~K}$ is shown in Fig. 7 for the first (upper sketch) and the second installation.

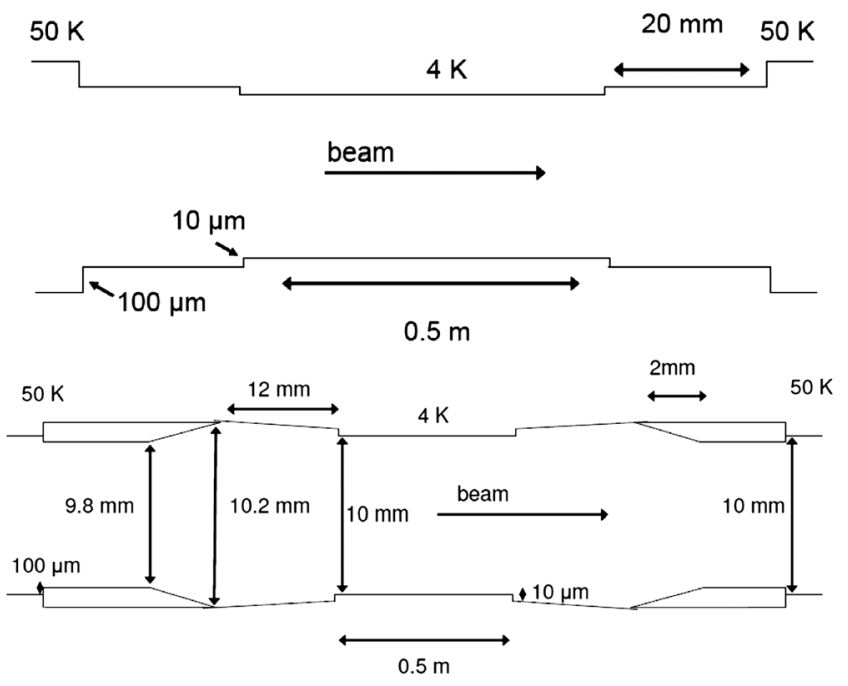

FIG. 7. Top: step transitions from the cold copper liner at $4 \mathrm{~K}$ to the $50 \mathrm{~K}$ shield made for the first installation of the COLDDIAG in the DLS. Bottom: tapered transitions from the cold copper liner at $4 \mathrm{~K}$ to the $50 \mathrm{~K}$ shield. 


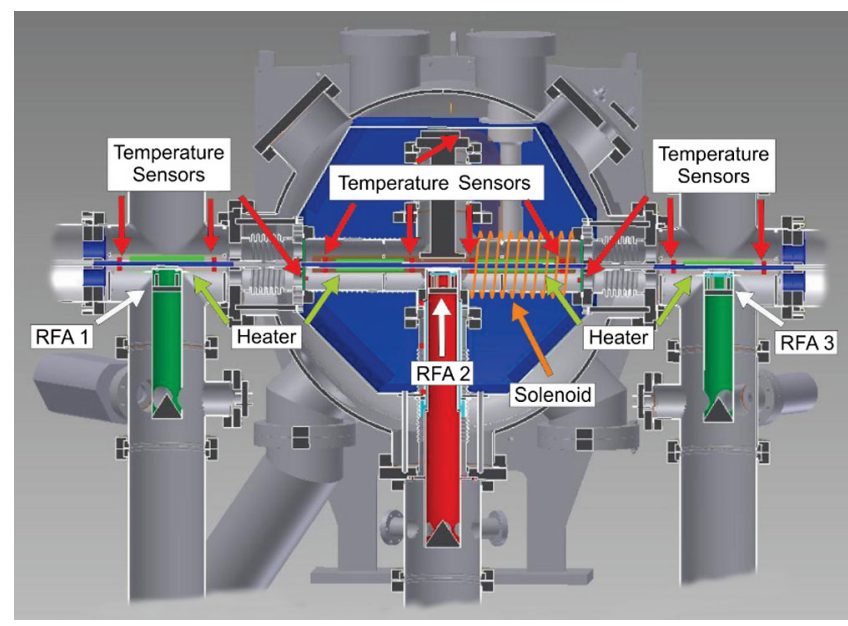

FIG. 8. Overview of the installed diagnostics devices in COLDDIAG.

\section{THE DIAGNOSTICS}

The diagnostic instrumentation of COLDDIAG comprises temperature sensors, pressure gauges, and residual gas analyzers as well as retarding field analyzers (see Fig. 8), which are presented in the following subsections.

\section{A. Temperature sensors and heaters}

COLDDIAG is equipped with 42 temperature sensors. Pt-100 sensors with a standard calibration curve [23] are used in the warm sections and in the cryostat, where an operating temperature higher than $50 \mathrm{~K}$ is expected. On the cold liner and everywhere else in the cryostat, Cernox sensors [A1] are used. A list of technical components used for diagnostics is given in Appendix A. The sensors located on the cryocooler have been calibrated by Lake Shore Cryotronics [24]. All other Cernox sensors have been calibrated at the Physikalisches Institut of the Karlsruhe Institute of Technology (KIT). As suggested by Lake Shore Cryotronics Inc., a Chebyshev polynomial of ninth order is fitted to the measured resistance values of the temperature sensors. The error of the calibration is less than $\pm 0.25 \mathrm{~K}$ in the region of interest (below $77 \mathrm{~K}$ ). There are six heaters in COLDDIAG: in each of the warm parts there is one on the top of the liner, while in the cold liner two are placed on the top and two on the bottom. The heaters simulate the heat intake from the beam to calibrate the measured temperature to a defined heating power. It is also possible to use these heaters to maintain a well-defined temperature and directly determine the heating power from the beam by the difference in power needed to stabilize the temperature with and without beam. The heaters are $120 \mathrm{~mm}$ long and $10 \mathrm{~mm}$ wide. They consist of a $0.125 \mathrm{~mm}$ diameter $99.99 \%$ pure $\mathrm{Ni}$-chrome wire clamped between a $1 \mathrm{~mm}$ thick thermal conducting sapphire plate and a $3 \mathrm{~mm}$ thermally isolating ceramic plate [A2]. Ni-chrome wire has the advantage of a high resistance, which changes very little compared with stainless steel during cool down [25], allowing one to have equivalent heaters in the warm and cold liner. With a resistance of $30 \Omega$ and a current of $0.5 \mathrm{~A}$, each one of the heaters can reach a maximum of around $7.5 \mathrm{~W}$. Each individual heater is controlled by a power supply [A3] with a maximum voltage of $60 \mathrm{~V}$ and a maximum current of 1.5 A. Four additional cartridge heaters [A4] with $50 \Omega$ resistance, are installed: two close to the shield at the 4 to $50 \mathrm{~K}$ transition between the liner and the cold cross in the UHV vacuum and two of them on the cold stage of the cryocooler head inside the insulation vacuum. These heaters can be used to apply larger amounts of heating power to the cryostat. The maximum base temperature applied to the cold liner during the installation in the DLS was $\simeq 35 \mathrm{~K}$. To obtain only the power dissipated by the heaters, a four-wire measurement technique is used. The voltage drop over each heater is measured with a multimeter [A5].

The wiring of the temperature sensors and heaters placed in the beam vacuum has to be radiation resistant, suitable for cryogenic use, and UHV compatible. The use of organic material in the beam vacuum has been avoided wherever possible. A nickel-copper wire [A6] with a thin insulation layer of sintered ceramic was used to connect the temperature sensors and heaters placed on the warm parts. To avoid noise pick up, the four wires of each sensor were twisted together. For the heaters and the temperature sensors on the cold liner, a phosphor bronze wire [A7] was chosen after vacuum and radiation testing. The wire is Formvar isolated and consists of two twisted pair wires, which are again twisted together, to minimize noise pickup. In total, five 50-pin feedthroughs, three from the cold section and one from each warm section, provide the signals to the outside of the vacuum chamber. To minimize noise pickup on the $35 \mathrm{~m}$ long cables from COLDDIAG to the control rack, all the cabling for the sensors is double shielded cable [A8]. As the voltage and the current are much higher in the heater circuits compared with the temperature sensor circuit, a cable without additional screening but of thicker cross section was chosen. A cross section of $0.5 \mathrm{~mm}^{2}$ is used for the power cable and a thinner $0.25 \mathrm{~mm}^{2}$ cross section is used for the sense circuit of the heaters [A9].

\section{B. Pressure gauges and residual gas analyzers}

Each of the three pumping ports contains a vacuum gauge [A10] and a residual gas analyzer [A11] to monitor the total pressure and the gas composition in the three sections of the UHV chamber. The lowest measurable pressure of the cold cathode gauges is below $10^{-10}$ mbar. The residual gas analyzer covers a mass span from 0-100 amu and is equipped with a Faraday cup and a continuous secondary electron multiplier (C-SEM) detector. The minimum measurable partial pressure is below $5 \times 10^{-12} \mathrm{mbar}$ with the Faraday cup and $10^{-14}$ mbar with the C-SEM detector. 


\section{Retarding field analyzers}

To measure the flux and the energy distribution of charged particles hitting the chamber walls, there is a retarding field analyzer (RFA) located at each of the pumping ports. The half moon shaped RFA in COLDDIAG consists of a tungsten mesh and a detector plate placed behind pumping holes in the beam tube (see Fig. 9). The beam tube is electrically grounded as it is connected to the cryostat. The tungsten mesh can be biased negatively or positively (against ground) with varying voltages. The detector plate can also be positively or negatively biased (against ground) opposite to the mesh to collect the charged particles passing through the repelling grid. The current of particles hitting the collector plate is measured by an electrometer [A12]. With the installed power supply [A13], a maximum retarding voltage of $250 \mathrm{~V}$ can be applied in steps as small as $10 \mathrm{mV}$. The collector voltage can be varied from 0 to $60 \mathrm{~V}$. Experiments

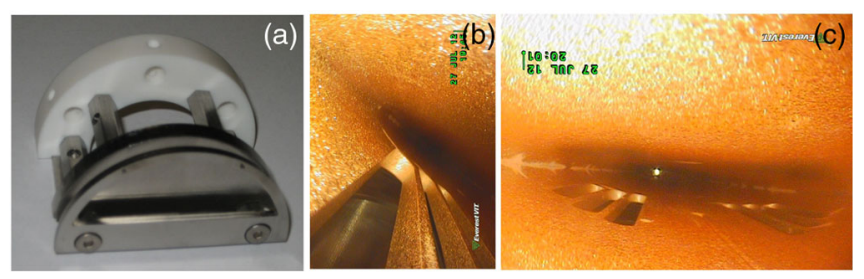

FIG. 9. Half-moon shaped RFA mounted into COLDDIAG without tungsten grid (a), the tungsten grid of the RFA under the pumping holes (b), and the pumping holes in the cold liner section (c).
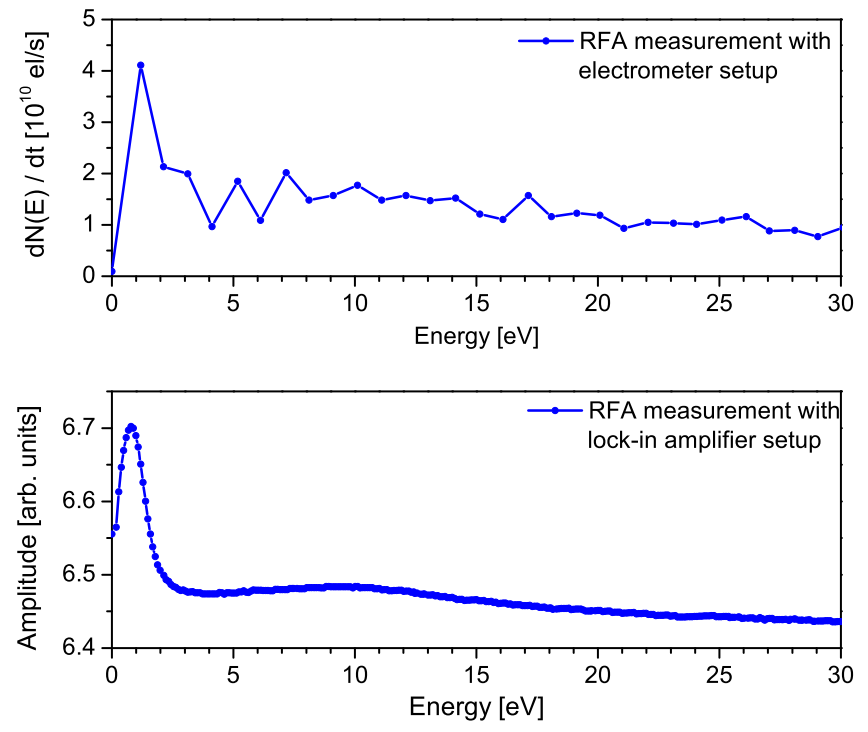

FIG. 10. Comparison of the two RFA readout setups. Energy spectrum obtained by the electrometer (top), and with the lock-in amplifier (bottom), of the low energy electrons hitting the detector and generated by a hot filament in a laboratory experiment. with a RFA with similar design and readout were performed at the ANKA storage ring. During the calibration done at the Laboratori Nazionali di Frascati (LNF) it appeared that the proposed RFA setup might not be sensitive enough to determine the energies of the primary electrons passing the retarding grid. Instead, the measured spectra are dominated by secondary electrons created at the grid or the collector [26]. Therefore, to improve the signal-to-noise ratio, a lockin technique was implemented in addition to the dc readout. A multiplexer [A14] is used to switch between the three RFA's and the two measurement options. A sinusoidal voltage supplied by the lock-in amplifier [A15] is coupled via a microphone transformer on top of the retarding voltage. A battery pack with a fixed voltage of $85 \mathrm{~V}$ is used to bias the collector. With the lock-in amplifier, the current on the collector is measured versus frequency and phase. A comparison between the two measurement modes done with a hot filament in a laboratory experiment was promising (see Fig. 10). In this test setup, the electron spectrum obtained with the electrometer showed already some noise with steps of $1 \mathrm{~V}$ on the retarding grid. On the contrary, the data measured by using the lock-in amplifier exhibited the expected broad spectrum and less noise even with an energy resolution of $0.1 \mathrm{eV}$.

\section{SOLENOID}

Over the downstream long arm of the cold six-way cross, a magnet coil is installed to suppress any charged particles impinging onto the liner walls. Low energy electrons with a velocity component perpendicular to the beam direction are deflected on a helical path and guided out of the cold liner section. The magnet coil has a length of $160 \mathrm{~mm}$ and an inner diameter of $120 \mathrm{~mm}$. To reach an on-axis peak field of $5 \mathrm{mT}$, a current of $0.43 \mathrm{~A}$ is needed. To prevent overheating, the coil is connected directly to the warm stage of the cryocooler and a sensor is installed on the windings to monitor the temperature. Whereas currents below $0.43 \mathrm{~A}$ can be applied for long periods, currents up to 1 A corresponding to a field of more than $10 \mathrm{mT}$ can be applied only for short times in the order of $30 \mathrm{~min}$. This is due to the slow heating up of the solenoid which impacts the temperature measurements.

\section{CALIBRATION}

In order to determine the beam heat load to the cold liner, a calibration of the temperature sensors is necessary. This is done using the heaters installed on the liner and the heaters installed at the upstream and downstream flanges connecting the $50 \mathrm{~K}$ shield through the thermal transitions to the cold part of the liner.

Two different approaches were used for the beam heat load measurements. In the first approach we observe the increase in temperature of the cooling connection from the cryocooler to the cold liner with beam. The increase in 


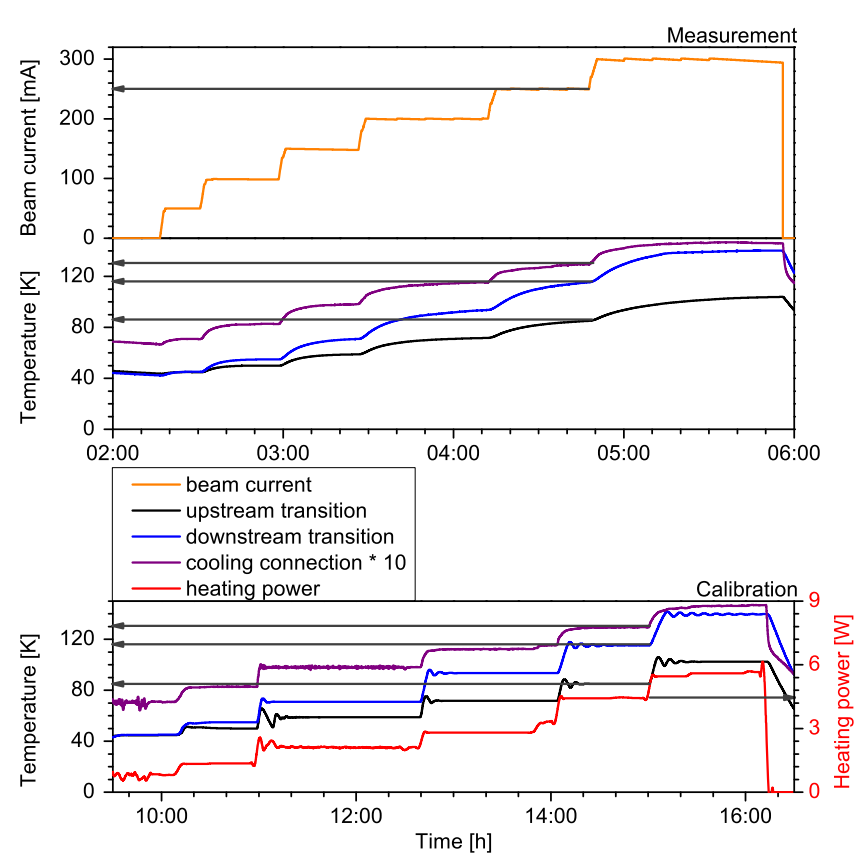

FIG. 11. Increase in temperature at the cooling connection used as a measure of the deposited beam heat load. Temperatures at cooling connection and transitions and heating power to the liner heaters during measurement (middle) and calibration (bottom) of a beam current ramp (top). The measurement is with 686 bunches.

temperature of the cooling connection is a measure of the deposited beam heat load on the whole liner. In the second approach the temperature of the cooling connection from the cryocooler to the cold liner is kept constant. The heaters on the liner are used to keep the cooling connection at a fixed temperature (e.g. $20 \mathrm{~K}$ ) during the measurements. The difference in power to the heaters with and without beam is equal to the beam heat load. The heaters installed at the upstream and downstream flanges of the $50 \mathrm{~K}$ shield can simulate the contribution of beam heating to the flanges transported to the cold liner by the thermal transitions. In order to determine the beam heat load to the cold liner, this contribution has been removed.

Examples of the calibration for a beam current ramp, measured by letting the temperature of the cooling connection increase and by keeping the temperature of the cooling connection constant, are shown in Figs. 11 and 12.

The points for the calibration are taken from the end of the single beam current steps; after that the cooling connection temperature (see Fig. 11) or the heating power (see Fig. 12) has adjusted to the beam changes and its further decrease is only depending on the increase in temperature at the thermal transitions. The calibration is performed without beam and reproduces the temperature distribution on the cooling connection and on the thermal transitions as observed at the above described measuring point. The difference between the heating power (red lines in Figs. 11 and 12) during one measurement and its respective calibration is the beam heat load.

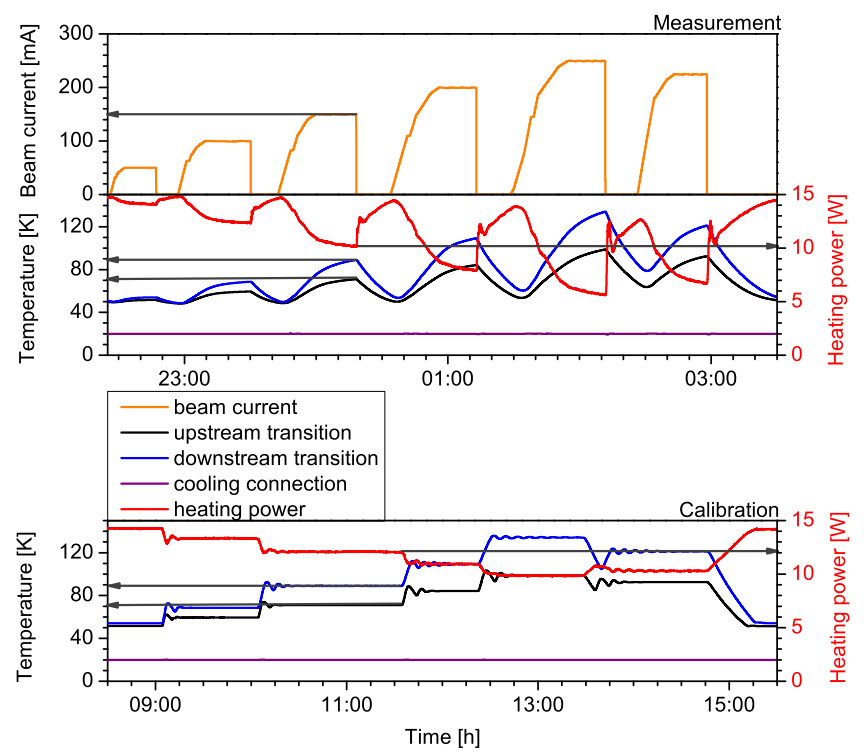

FIG. 12. Constant temperature at the cooling connection. Temperatures at cooling connection and transitions and heating power to the liner heaters during measurement (middle) and calibration (bottom) of a beam current ramp (top). The measurement is with 460 bunches.

For the warm sections we observe the increase in average temperature of the liner sensors and use the heaters on the warm liner for calibration. Possible additional influences from heat transported from the connections of the flanges to the warm liners are included in the measured values. Since no temperature sensors on the flanges connecting the warm liner to the external pipe were fitted, it is not possible to distinguish the beam heat load to the warm liners from the one deposited in the connections to the flanges. This can be improved in the future. The time constant to reach thermal equilibrium in the warm sections is much longer (about $48 \mathrm{~h}$ ) than in the cold one, because of the absence of active cooling. We could therefore measure the beam heat load to the warm liners during constant beam conditions over a few days, in user operation. Because of a thermal contact problem in the downstream warm section, which can be solved for future installations, only the beam heat load to the upstream warm liner could be measured.

The estimated error on the beam heat load is within $0.1 \mathrm{~W}$.

\section{ALIGNMENT}

During the installation of COLDDIAG in the DLS storage ring, the cryostat and the upstream and downstream pumping stations, which connect COLDDIAG to the storage ring, were positioned with respect to the nominal electron beam orbit, using a laser tracker to measure the position of the $\mathrm{CF}$ flanges. The inserted components end at the entrance and at the exit with two gate valves. Starting from the left, Fig. 13 shows a pumping station, a taper, 

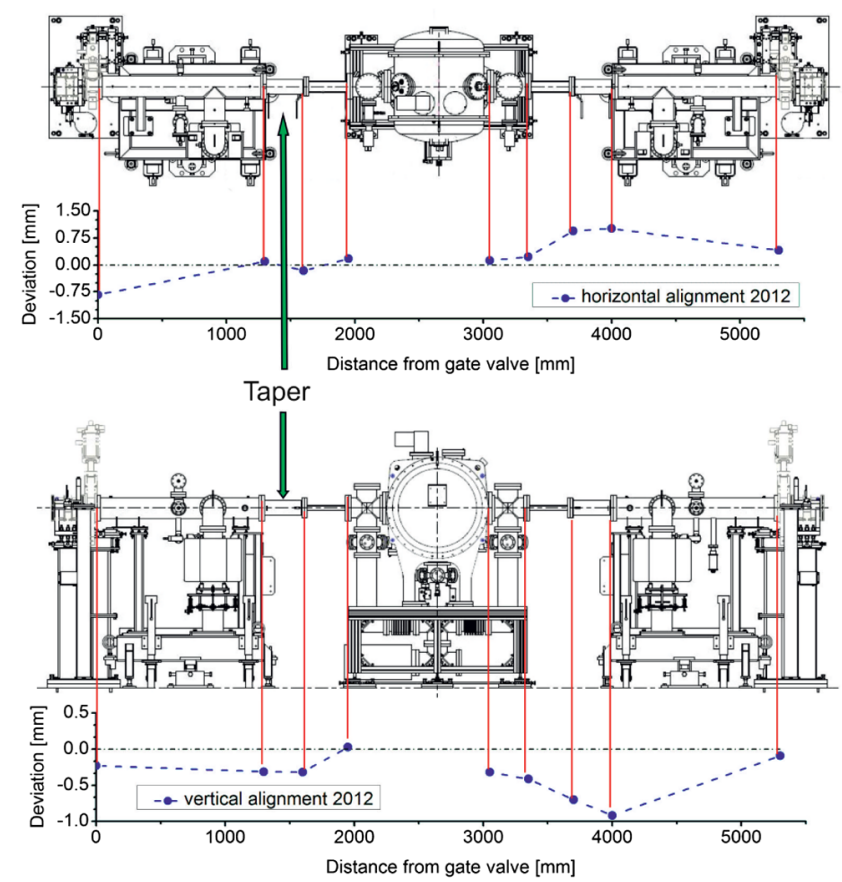

FIG. 13. Measurement of the position of the components in the COLDDIAG straight. The upper picture shows the horizontal alignment, the bottom one the vertical alignment.

acting also as a radiation mask to completely screen the warm and cold liner of COLDDIAG from direct synchrotron radiation from the upstream bending magnets, COLDDIAG including the warm parts, a second taper, and a second pumping station. Figure 13 shows the horizontal and vertical position of the components in the straight section where COLDDIAG is installed. The horizontal position of the upstream taper and COLDDIAG is within $\pm 250 \mu \mathrm{m}$ of the nominal beam axis. The vertical position has only a small negative offset of around $300 \mu \mathrm{m}$ compared to nominal beam position. The deviations in the nominal position of the gate valves and the downstream components can be tolerated because of the larger beam aperture of these components. The vertical deviation of the liner has been measured with a Leica NA2 level and it is within about $\pm 270 \mu \mathrm{m}$ from the nominal position given by a line defined by the end flanges of the rf bellows, which were leveled before the measurement. A Leica NA2 level and a Polyether ether ketone optical target have been used to measure any movement of the liner during cool down. Between the first and second installation the vertical movement of the liner during cool down has been reduced from $700 \mu \mathrm{m}$ (upwards) to less than $50 \mu \mathrm{m}$. This has been achieved by changing the connection between the liner, the six-way cross, and the $50 \mathrm{~K}$ shield. The liner movement during the first installation has also caused a plastic deformation in the rf fingers placed in the bellows, bending them. Before the second installation the rf fingers were bent back by adding a clamp, which reinforces the electrical contact.

\section{FIRST RESULTS}

In order to show the capabilities of COLDDIAG we present here first results obtained at the DLS.

In Fig. 14 some of the beam heat load measurements for different beam current ramps are shown. No significant difference is observed between the measurements performed keeping the liner (cooling connection) at a constant temperature of $20 \mathrm{~K}$ and letting the liner (cooling connection) increase in temperature by beam heating (see also Sec. VI). A significant discrepancy is observed from the beam heat load values predicted by heating due to geometrical and resistive wall impedance [27]. The warm sections, which are about half as long as the cold section, exhibit about the same beam heat load.

Figure 15 shows the pressure on the gauges located in the straight section upstream, downstream, and in COLDDIAG as a function of the beam current. The measurement was performed with $3 \mathrm{GeV}$ and 460 consecutive bunches separated by $2 \mathrm{~ns}$. The pressure measured in the upstream pumping section has a linear dependence on the beam current, in contrast to the pressure measured with the other gauges. This linear behavior is typical for photodesorption. The residual gas measured in all three sections exhibits an analog composition dominated by hydrogen with the main peak at 2 amu and followed by water indicated by the peak at $18 \mathrm{amu}$. The presence of water is due to the fact that COLDDIAG is not baked. Baking at higher temperatures than $70^{\circ} \mathrm{C}$ is not possible without damaging the cryocooler and the thermal connections in UHV where indium is used. Baking has not been performed to avoid any influence on the purity of the electroplated copper layer on the liner. In Fig. 16 a comparison of the gas content with and without

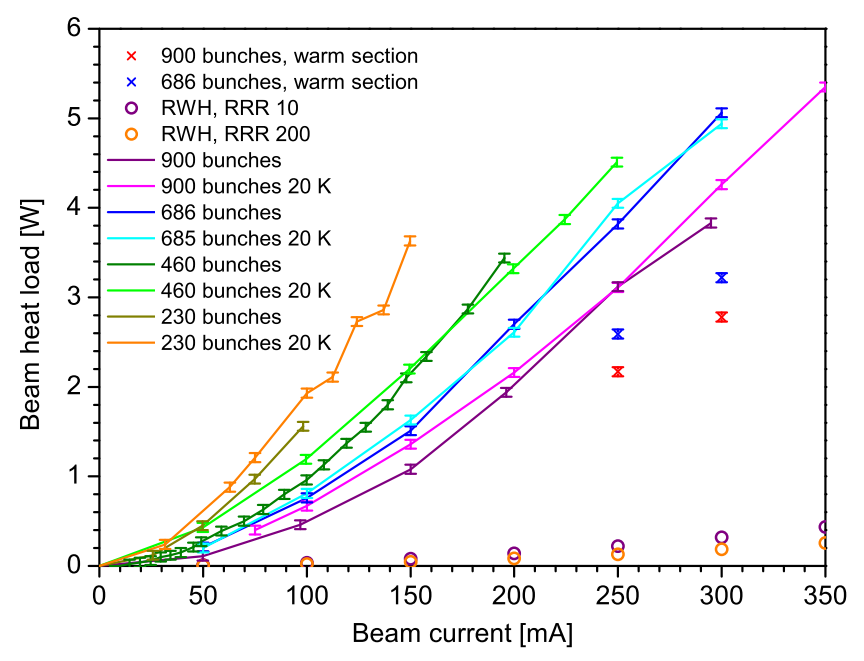

FIG. 14. Beam heat load displayed as a function of beam current corrected by the offline calibration. The circles show the expected beam heat load from resistive wall heating for two different purities of copper. The crosses show the beam heat load measured in one of the warm sections. Measurements at constant liner temperature are indicated with $20 \mathrm{~K}$. 


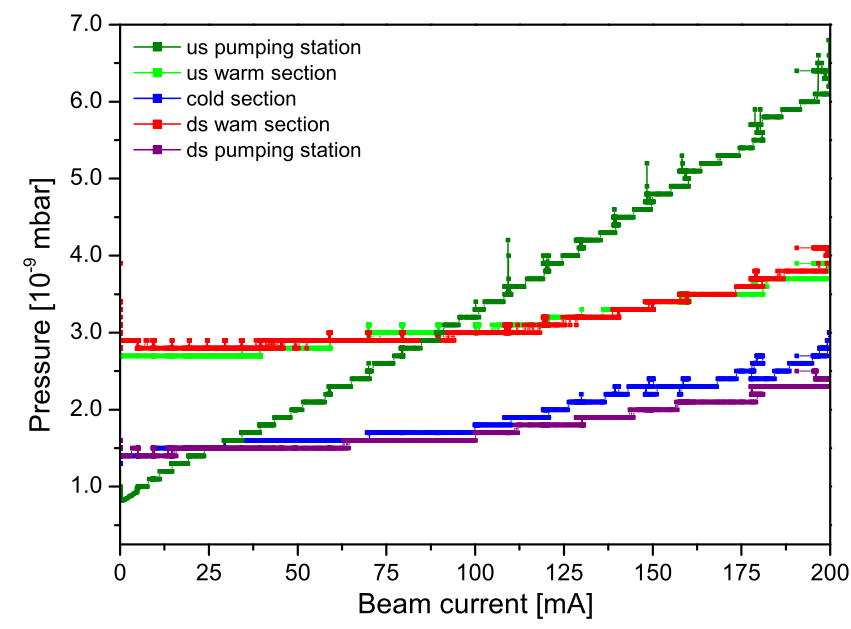

FIG. 15. Pressure in the COLDDIAG straight section as a function of beam current, with a fill pattern of 460 consecutive bunches.
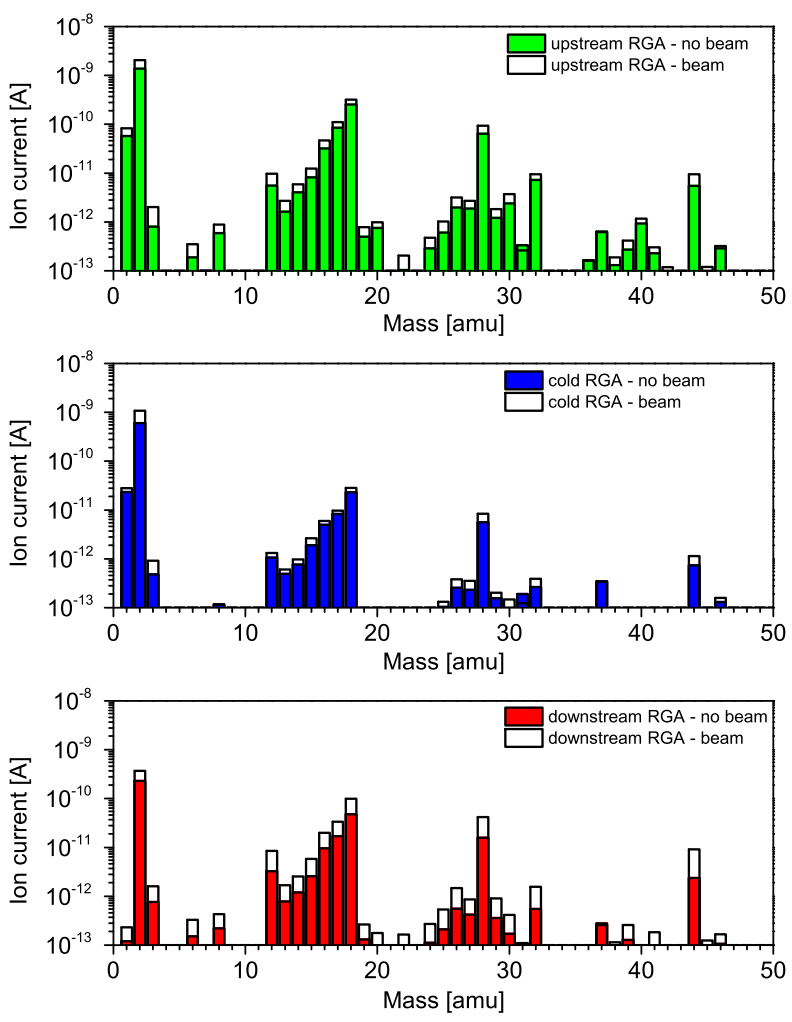

FIG. 16. Mass spectra measured with the three RGAs installed in the upstream, downstream, and cold section of COLDDIAG, with and without beam.

beam shows that the partial pressure of all gases increases almost equally and no particular gas is accumulating.

In order to distinguish whether ions or electrons are hitting the wall, while the retarding grid of the RFAs was connected to ground, the collector plate voltage was varied from negative to positive values. The measurements show that most of the particles hitting the wall have a negative charge.
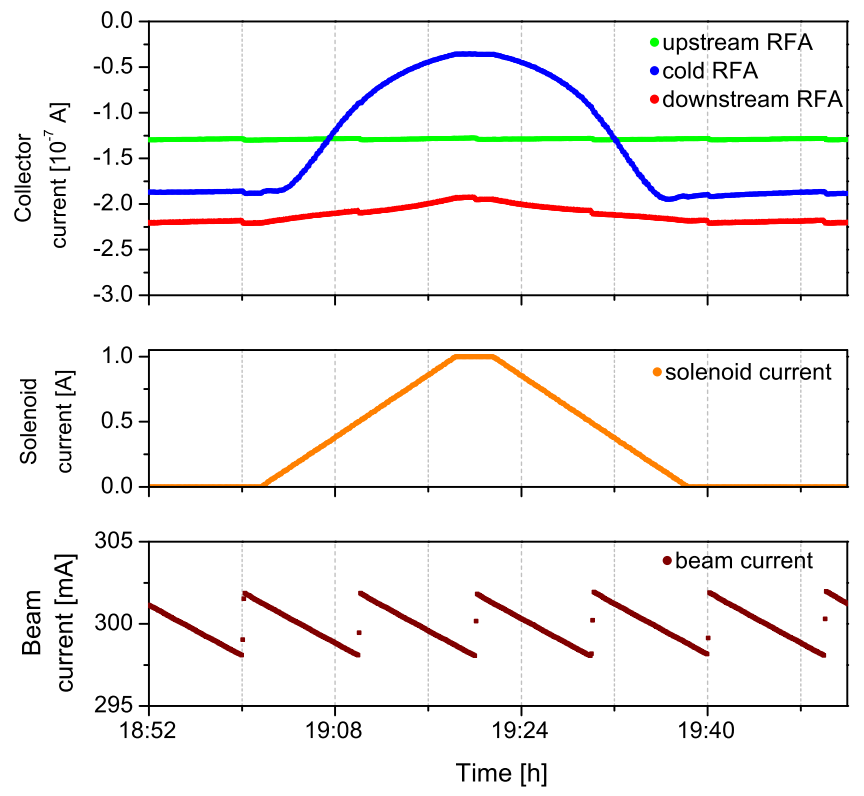

FIG. 17. Influence of the axial magnetic field produced by the solenoid on the particle flux. The collector is positively biased with $+50 \mathrm{~V}$.

Figure 17 shows the influence of the solenoid on the flux of the low energy electrons impinging the wall with positive $(+50 \mathrm{~V})$ collector voltage. The current on the upstream RFA is not changed by changing the magnetic field.

\section{CONCLUSIONS AND OUTLOOK}

We have presented here a unique instrument to measure the beam heat load to cold and warm bores equipped with additional diagnostics to measure the pressure, gas content, and the flux of charged particles impinging the wall.

We have described the vacuum and cryogenic layouts, the implemented diagnostics, as well as the calibration and the alignment of COLDDIAG in the DLS.

Examples of first results obtained with COLDDIAG at the DLS are shown to demonstrate the capabilities of this instrument.

Experimental and theoretical work is ongoing, with the aim of explaining the very high measured beam heat load compared with existing theories. A future paper will be dedicated to present an overview of all the measurements performed with COLDDIAG at the DLS and their detailed analysis.

\section{ACKNOWLEDGMENTS}

The authors would like to thank J. Clarke (STFC/ Daresbury) for kindly providing pumps and pressure gauges, N. Glamann (KIT) for valuable technical support, E. Wallén (MAXLab) for useful discussions at the beginning of the project, R. Cimino (LNF), M. Commisso (LNF), and R. Weigel (Bruker GmbH) for their help with the retarding field analyzers and, V. Baglin (CERN) and 
G. Bregliozzi (CERN) for the calibration of the pressure gauges and residual gas analyzers, and Prof. H.v. Löhneysen and his group (KIT) for supplying the setup used for the calibration of the temperature sensors. We would also like to thank T. Baumbach (KIT) and M. Hagelstein (KIT) for supporting the project. This project was supported by the program PNI of the Helmholtz Association.

\section{APPENDIX A}

Following is a list of technical components used for diagnostics.

1. Lakeshore Cryotronics Inc. Cernox 1050SD

2. MACOR machinable ceramic

3. Thurbly Thandar Instruments PL601-P

4. Lake Shore Cryotronics Inc. HTR-50

5. Keithley Instruments Inc. Model 2700 multimeter equipped with Model 770020 channel differential multiplexer module [28]

6. Karl Schupp ceramic wire KD 500 AWG 32

7. Lakeshore Quad-Twist wire AWG 36

8. Lappkabel UNITRONIC LAN 1200 H CAT.7 halogen-free

9. Lappkabel UNITRONIC LiHCH (TP) halogenfree

10. MKS Instruments Inverted Magnetron Gauge

11. Pfeiffer Vacuum PrismaPlus QMG 220 [29]

12. Keithley Instruments Inc. Model 6514

13. Thurbly Thandar Instruments PLH250-P

14. Signal Recovery 3830 BNC multiplexer [30]

15. Signal Recovery 7230 dual phase lock-in amplifier.

[1] N. Mezentsev and E. Wallén, Synchrotron Radiat. News 24, 3 (2011).

[2] E. Wallén and G. LeBlanc, Cryogenics 44, 879 (2004).

[3] J. C. Schouten and E. C. M. Rial, Proceedings of the 2nd International Particle Accelerator Conference, San Sebastián, Spain (EPS-AG, Spain, 2011).

[4] S. Casalbuoni, M. Hagelstein, B. Kostka, R. Rossmanith, M. Weisser, E. Steffens, A. Bernhard, D. Wollmann, and T. Baumbach, Phys. Rev. ST Accel. Beams 9, 010702 (2006).

[5] S. Casalbuoni, A. Grau, M. Hagelstein, R. Rossmanith, F. Zimmermann, B. Kostka, E. Mashkina, E. Steffens, A. Bernhard, D. Wollmann, and T. Baumbach, Phys. Rev. ST Accel. Beams 10, 093202 (2007).

[6] A.-S. Müller, I. Birkel, B. Gasharova, E. Huttel, R. Kubat, Y.-L. Mathis, D. A. Moss, W. Mexner, R. Rossmanith, M. Wuensch, P. Wesolowski, F. Pérez, M. Pont, and C. J. Hirschmugl, in Proceedings of the 21st Particle Accelerator Conference, Knoxville, 2005 (IEEE, Piscataway, NJ, 2005), p. 2518.

[7] S. Casalbuoni, S. Gerstl, A. Grau, T. Holubek, and D. Saez de Jauregui, in Proceedings of the 3rd International Particle Accelerator Conference, New Orleans, LA, 2012 (IEEE, Piscataway, NJ, 2012), p. 717.
[8] S. Casalbuoni, S. Schleede, D. Saez de Jauregui, M. Hagelstein, and P. F. Tavares, Phys. Rev. ST Accel. Beams 13, 073201 (2010).

[9] S. Prestemon, https://accelconf.web.cern.ch/accelconf/ FEL2011/talks/thoai2_talk.pdf.

[10] F. Trillaud, S. Prestemon, R. D. Schlueter, and S. Marks, IEEE Trans. Appl. Supercond. 21-3, 1756 (2011).

[11] S. Casalbuoni, T. Baumbach, A. Grau, M. Hagelstein, R. Rossmanith, V. Baglin, B. Jenninger, R. Cimino, M. Cox, E. Mashkina, and E. Wallén, in Proceedings of the 11th European Particle Accelerator Conference, Genoa, 2008 (EPS-AG, Genoa, Italy, 2008), p. 2240.

[12] S. Casalbuoni, T. Baumbach, S. Gerstl, A. Grau, M. Hagelstein, D. Saez de Jauregui, C. Boffo, G. Sikler, V. Baglin, R. Cimino, M. Commisso, B. Spataro, A. Mostacci, M. Cox, J. Schouten, E. Wallén, R. Weigel, J. Clarke, D. Scott, T. Bradshaw, I. Shinton, and R. Jones, IEEE Trans. Appl. Supercond. 21-3, 2300 (2011).

[13] S. Gerstl, T. Baumbach, S. Casalbuoni, A. Grau, M. Hagelstein, T. Holubek, D. Saez de Jauregui, V. Baglin, C. Boffo, G. Sikler, T. Bradshaw, R. Cimino, M. Commisso, A. Mostacci, B. Spataro, J. Clarke, R. Jones, D. Scott, M. Cox, J. Schouten, I. Shinton, E. Wallén, and $\mathrm{R}$. Weigel, in Proceedings of the 2nd International Particle Accelerator Conference, San Sebastián, Spain (EPS-AG, Spain, 2011), p. 3269.

[14] J. Cui, J.P. Xu, W. Li, M. Li, Z.C. Zhang, X. K. Ji, S. Prestemon, T. Koettig, R. Schlueter, P. Zhang, and Y. Cheng, IEEE Trans. Appl. Supercond. 24-3, 0503604 (2014).

[15] S. Gerstl, T. Baumbach, S. Casalbuoni, A. Grau, M. Hagelstein, T. Holubek, D. Saez de Jauregui, R. Bartolini, M.P. Cox, J.C. Schouten, R. Walker, M. Migliorati, B. Spataro, and R. R. Shinton, in Proceedings of the 3rd International Particle Accelerator Conference, New Orleans, LA, 2012 (IEEE, Piscataway, NJ, 2012), p. 720 .

[16] S. Gerstl, S. Casalbuoni, A. Grau, T. Holubek, D. Saez de Jauregui, R. Voutta, R. Bartolini, M. P. Cox, E. C. Longhi, G. Rehm, J. C. Schouten, R. Walker, M. Migliorati, and B. Spataro, in Proceedings of the 4th International Particle Accelerator Conference, IPAC-2013, Shanghai, China, 2013 (JACoW, Shanghai, China, 2013), p. 2135.

[17] M. Furman, H. Lee, and B. Zotter, SSC-116, 1987, http://lss .fnal.gov/archive/other/ssc/ssc-116.pdf; E. Métral, Proceedings of Chamonix 2012 Workshop on LHC Performance, edited by C. Carli (CERN, Geneva, 2012), pp. 105-115.

[18] C. Boffo, W. Walter, T. Baumbach, S. Casalbuoni, S. Gerstl, A. Grau, M. Hagelstein, and D. Saez de Jauregui, IEEE Trans. Appl. Supercond. 21-3, 1756 (2011).

[19] Y. Ivanyushenkov and E. Moog, Synchrotron Radiat. News 24-3, 20 (2011).

[20] V. Baglin, I. R. Collins, and B. Jenninger, Vacuum 73, 201 (2004).

[21] SHI Cryogenics Group, http://www.shicryogenics.com.

[22] S. Gerstl, Ph.D. thesis, Karlsruhe Institute of Technology, Karlsruhe, Germany, 2013.

[23] Pt100 calibration. 
[24] Lake Shore Cryotronics, Inc., http://www.lakeshore.com.

[25] J. E. Jensen, R. B. Stewart, and W. A. Tuttle, Selected Cryogenic Data Notebook (Brookhaven National Laboratory, Bubble Chamber Group, 1966).

[26] D. Saez de Jauregui, S. Casalbuoni, A. Grau, M. Hagelstein, R. Cimino, M. Commisso, E. Mashkina, and R. Weigel, in Proceedings of the 23rd Particle Accelerator
Conference, Vancouver, Canada, 2009 (IEEE, Piscataway, NJ, 2009), p. 330.

[27] S. Casalbuoni, M. Migliorati, A. Mostacci, L. Palumbo, and B. Spataro, JINST 7, P11008 (2012).

[28] Tektronix GmbH. Keithley Instruments Inc., 2013, http:// www.keithley.de/.

[29] Pfeiffer Vacuum GmbH, http://www.pfeiffer-vacuum.de.

[30] AMETEK Inc., 2013, http://www.signalrecovery.com/. 